www.nature.com $/ \mathrm{hr}$

\title{
CRP induces hypertension in animal models: homo sapiens says NO
}

\author{
Ishwarlal Jialal, Sridevi Devaraj and David Siegel \\ Hypertension Research (2011) 34, 801-802; doi:10.1038/hr.2011.59; published online 19 May 2011
}

$\mathrm{C}$ -reactive protein (CRP) is a prototypic downstream marker of inflammation. Recent data have suggested that CRP is an independent risk marker for cardiovascular disease. In addition, numerous lines of evidence support the thesis that CRP could participate in atherothrombosis. ${ }^{1}$ In both in-vitro and in-vivo studies, one of the most uniform findings is the inhibition of endothelial nitric oxide synthase (eNOS) and impaired endothelial vasoreactivity, following CRP administration. ${ }^{2}$

Epidemiological studies support a relationship between higher levels of CRP predicting hypertension. ${ }^{3}$ In this issue of the journal, $\mathrm{Li}$ et al. ${ }^{4}$ show that delivery of CRP via a single injection of adeno-associated virus (AAV) over expression of human CRP (AAVhCRP) vs. AAV-green fluorescent protein results in an increase of CRP upto $25 \mathrm{mg} \mathrm{l}^{-1}$ in Sprague-Dawley rats. Coupled with this increased CRP, they show an increase in both systolic and intra-arterial blood pressure (BP). In an attempt to elucidate the molecular mechanisms, they show an increase in reactive oxygen species possibly due to increased NADPH oxidase activity and decreased SOD1, decreased eNOS and an increase in Rho kinase. All these suggested mechanisms could result in an increase in hypertension. This study follows a previous report in which Guan et al. ${ }^{5}$ reported that a single injection of AAV-hCRP into male Wistar rats resulted in efficient sustained expression of CRP in the liver, together with an increase in serum CRP to $15 \mathrm{mgl}^{-1}$ at $2-4$ months compared with AAV-green fluores-

Dr I Jialal and Dr D Siegel are at the VA Medical Center, Mather, CA, USA and Dr I Jialal and Dr S Devaraj are at the Department of Pathology \& Laboratory Medicine, University of California at Davis Medical Center, Sacramento, CA, USA.

E-mail: ishwarlal.jialal@ucdmc.ucdavis.edu cent protein. Most importantly in this paper, they also showed that human CRP resulted in an increase in systolic and mean arterial pressure. Collectively in these papers, results showed that in addition to impaired vasoreactivity, there was an increase in the expression of angiotensin-1 (AT-1) receptors, endothelin-1 and the ETA type-1 receptors. They also showed a decreased expression of eNOS and AT-2 receptors. This was accompanied by impairment in endotheliumdependent vasorelaxation.

Previously, Vongpatanasin et al. ${ }^{6}$ reported that CRP induces hypertension in CF1 transgenic mice expressing rabbit CRP (CF1-CRP) under the regulation of the phosphoenolpyruvate carboxykinase promoter, compared with wild-type CF1 control. CRP levels attained were about $10-15 \mathrm{mgl}^{-1}$. It should be emphasized, however, that unlike the current study and the study by Guan et al., ${ }^{5}$ they suggested this effect was not attributable to the effect of theAT-1 receptor, which was unaltered, but to a decrease in the AT-2 receptor. However, the study by Vongpatanasin et al. ${ }^{6}$ provided little further information with regard to molecular insights.

Recently, Pravanec et al. ${ }^{7}$ reported that in spontaneously hypertensive rats, human CRP under the control of the apolipoprotein $\mathrm{E}$ promoter resulted in a substantial increase in human CRP levels. This increase in CRP resulted in an increase in both systolic and diastolic BPs measured by telemetry, compared with the spontaneously hypertensive rat controls. They suggest in their paper that CRP resulted in induction in many features of the metabolic syndrome. Transgenic spontaneously hypertensive rats exhibited insulin resistance, hypertriglyceridemia, hypertension, reduced serum adiponectin and microalbuminuria. Transgenic sponta- neously hypertensive rats had evidence of inflammation and oxidative tissue damage with increased serum levels of interleukin 6 and increased hepatic and renal thiobarbituric acid-reactive substances, suggesting that oxidative stress may be mediating adverse effects of increased human CRP. A major critique of this manuscript is that human CRP levels attained in the study of $250 \mathrm{mgl}^{-1}$ are clearly not what is seen in patients with metabolic syndrome or hypertension. Rather, it is more akin to levels seen in patients with macroinflammation, such as bacterial infections and so on. Thus, the relevance of these findings with regard to hypertension and metabolic syndrome is questionable. However, the reports of the Vongpatanasin and the Wang group convincingly demonstrate that induction of CRP levels upto $25 \mathrm{mgl}^{-1}$ results in a significant increase in BP.

Thus, it appears the CRP could induce hypertension in three different animal models through various molecular mechanisms (potential mechanisms with CRP that could culminate in hypertension are depicted in Figure 1). These include an increase in ET-1, a decrease in eNOS and vasoreactivity, an increase in reactive oxygen species, modulation of AT receptors, and a decrease in prostacyclin, a potent vasodilator. This latter mechanism was not studied by any of the previous groups but was previously reported by Venugopal et al. ${ }^{8}$ Also, recently it was shown that CRP can in the presence of aldosterone promote endothelial stiffness. ${ }^{9}$

The paper by $\mathrm{Li}$ et $a .^{4}$ goes on to show that the use of rosuvastatin results in a reduction in CRP-induced hypertension compared with controls. They also show that the use of rosuvastatin ameliorated the deleterious effects of CRP and advanced this as a 


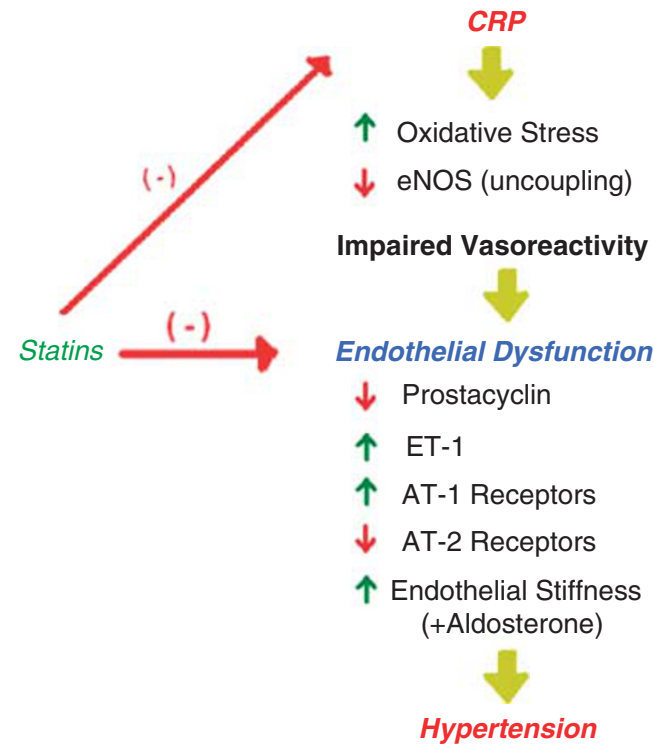

Figure 1 Mechanism(s) via which CRP induces Hypertension.

plausible mechanism to explain the increase in BP without lowering CRP. This contrasts with results in humans in whom statins as a class lower CRP. ${ }^{10}$ The three groups suggest that CRP can indeed induce hypertension. An underpinning of the induced hypertension is the inhibition of eNOS and impaired vasoreactivity. The translational and clinical relevance of these findings, especially with regard to the decrease in BP with rosuvastatin, which was not normalized, has potential clinical ramifications. In the JUPITER study, rosuvastatin lowered CRP but did not lower BP; however, this was not carefully monitored as it was not a prespecified end point. ${ }^{3}$

If a study in humans can demonstrate that CRP-induced hypertension can be ameliorated with antisense therapy or other therapies that lower CRP (such as statins), or small molecular inhibitors of CRP, this will constitute proof of the principle that CRP causes hypertension Although we await these studies, what is clinically relevant immediately, and as Wang's group argued, is the use of a statin in patients who have refractory hypertension. Statins, by improving vasoreactivity and endothelial function, could facilitate better BP control when combined with antihypertensive strategies, such as those that target the renin-angoitensin-aldosterone system. These include AT receptor blockers, AT-converting enzyme inhibitors, and aldosterone and renin inhibitors.

However, as these three studies were conducted in animal models, the immediate clinical relevance remains uncertain. Collectively, these studies suggest that CRP could participate in the pathogenesis of hypertension through induction of oxidative stress and endothelial dysfunction underscoring the role of inflammation in the genesis of hypertension. It will be interesting to determine in human studies whether the introduction of a statin in a patient with refractory hypertension results in better BP control compared with a patient on antihypertensive therapy alone. Statins have been shown to have modest BP-lowering effect. ${ }^{3}$

\section{CONFLICT OF INTEREST}

The authors declare no conflict of interest.

\section{ACKNOWLEDGEMENTS}

This work was supported by NIH RO1 HL74360. We thank Kimberly Pierson for manuscript preparation.

1 Devaraj S, Singh U, Jialal I. The evolving role of C-reactive protein in atherothrombosis. Clin Chem 2009; 55: 229-238.

2 Jialal I, Verma S, Devaraj S. Inhibition of endothelial nitric oxide synthase by C-reactive protein: clinical relevance. Clin Chem 2009; 55: 206-208.

3 Devaraj S, Siegel D, Jialal I. Statin therapy in metabolic syndrome and hypertension post-JUPITER: what is the value of CRP? Curr Atheroscler Rep 2011; 13: 31-42.

4 Li X, Yang G, Zhao G, Wu B, Edin ML, Zeldin DC, Wang DW. Rosuvastatin attenuates the elevation in blood pressure induced by overexpression of human C-reactive protein. Hypertens Res 2011; 34: 869-875.

5 Guan H, Wang P, Hui R, Edin ML, Zeldin DC, Wang DW. Adeno-associated virus-mediated human $\mathrm{C}$-reactive protein gene delivery causes endothelial dysfunction and hypertension rats. Clin Chem 2009; 55: 274-284.

6 Vongpatanasin W, Thomas GD, Schwartz R, Cassis LA, Osborne-Lawerence S, Hahner L, Gibson LL, Black S, Samols D, Shaul PW. C-reactive protein causes downregulation of vascular angiotensin subtype 2 receptros and systolic hypertension in mice. Circulation 2007; 115: 1020-1028.

7 Pravanec M, Kajiya T, Zidek V, Landa V, Mlejnek P, Simakova M, Silhavy J, Malinksa $H$, Oliyarnyk $O$, Kazdova L, Fan J, Wang J, Kurtz T. Effects of human C-reactive protein on pathogenesis of features of the metabolic syndrome. Hypertension 2011; 57: 731-737.

8 Venugopal SK, Devaraj S, Jialal I. CRP decreases prostatcyclin release from human aortic endothelial cells. Circulation 2003; 108: 1676-1678.

9 Kusche-Vihrog K, Urbanova K, Blanqué A, Wilhelmi M, Schillers $H$, Kliche K, Pavenstädt $H$, Brand $E$, Oberleithner $H$. C-reactive protein makes human endothelium stiff and tight. Hypertension 2011; 57: 231-237.

10 Jialal I, Stein D, Balis D, Grundy SM, Adams-Huet B, Devaraj S. Effect of hydroxymethyl glutaryl coenzyme a reductase inhibitor therapy on high sensitive C-reactive protein levels. Circulation 2001; 103: 1933-1935. 